\title{
The Detection of Ultrasound Using Fiber-Optic Sensors
}

\author{
Brian Culshaw, Graham Thursby, Daniel Betz, and Borja Sorazu
}

\begin{abstract}
Ultrasound is a valuable tool for the detection of damage in structures and the characterization of material properties. Its detection is conventionally done by piezoelectric transducers, however fiber-optic sensors can operate over a greater range of frequencies and also yield information on the direction of wave propagation. The interaction between fiber sensors and ultrasound both demonstrates the integrating features of intrinsic fiber-optic sensors and presents new opportunities in ultrasonic detection, offering enormous diversity in polar and frequency response. This paper summarizes the interaction mechanisms between ultrasound and fiber sensors and confirms their functional flexibility. We use these results to demonstrate the practical use of these sensors to detect and locate damage in a sample.
\end{abstract}

Index Terms-Bragg grating, damage detection, polarimeter, ultrasound.

\section{INTRODUCTION}

D ETECTING ultrasound using intrinsic fiber-optic sensors is a topic which has been studied spasmodically over many years though, despite the large number of papers published on fiber Bragg grating (FBG) applications, until recently relatively little had been written on the subject. Fisher et al. [1] demonstrated the feasibility of using short fiber Bragg gratings to measure megahertz acoustic fields and temperature simultaneously. Formitchov [2] demonstrated the application of FBGs to measure ultrasonic pressure waves and proposed applications in measuring ultrasound in liquids and solids, while an underwater acoustic sensor using FBGs was described by Takahashi [3] using a tunable laser to interrogate the laser. A theoretical analysis of the use of FBGs for ultrasound detection was presented by Coppola [4], which focussed on the effect of the ultrasonic strain field on the grating response. More recently Lamb wave measurement for damage detection in composite materials has been described by Tsudu [5] and Takeda [6]

The interaction between and ultrasonic wave and a fiber is in principle very simple - the fiber integrates the effect of the ultrasound along the predetermined interaction length. However, this

Manuscript received August 31, 2007; revised November 16, 2007; accepted November 16, 2007. Published July 16, 2008 (projected). The associate editor coordinating the review of this paper and approving it for publication was Prof. Ignacio Matias.

B. Culshaw and G. Thursby are with the University of Strathclyde, Glasgow G1 1XW, U.K. (e-mail: b.culshaw@eee.strath.ac.uk; g.thursby@eee.strath.ac. uk).

D. Betz is with Daimler-Chrysler AG, 71059 Sindelfingen, Germany (e-mail: daniel.betz@daimlerchrysler.com).

B. Sorazu was with the University of Strathclyde, Glasgow G1 1XW, U.K. $\mathrm{He}$ is now with the University of Glasgow, Glasgow G128QQ, U.K. (e-mail: b.sorazu@physics.gla.ac.uk).

Color versions of one or more of the figures in this paper are available online at http://ieeexplore.ieee.org.

Digital Object Identifier 10.1109/JSEN.2008.927240 simple observation conceals a rich variety of sensing options in terms of both frequency and polar response dependent predominantly on the ratio of the interaction length to the ultrasonic wavelength and the mechanical field (strain, pressure or displacement) to which the sensor is configured to respond. This contrasts very strongly with the piezoelectric ceramic detectors which are the mainstay of conventional ultrasonic systems. In particular, the responses of piezo ceramic detectors are often dominated by mechanical resonances within the frequency range of the ultrasound to be detected. In contrast fiber sensors typically show no such resonant behavior at least until a few tens of megahertz when the first radial resonances in the fiber itself begin to appear. All the work we describe here and indeed much of the activity in ultrasonic testing and evaluation is at frequencies well below these initial resonance phenomena. Other frequency dependent effects may, however, be important as will be described later. Another very significant difference between the responses of fiber sensors and PZT disc sensors is that the former are heavily dependent on the angle between the direction of propagation of the wave and the fiber axis. The remainder of this paper first considers the integrating properties of optical fibers sensors in ultrasonic fields as a function of this angle. We then continue to discuss the implications of these interactions in sensor systems with long and short (compared to the ultrasonic wavelength) interaction lengths. The practical exploitation of the directional properties of the sensors to determine not only the amplitude, but also the direction of an incident wave is described. Finally one illustration of the potential use of such interactions in damage and detection location systems is presented.

\section{INTEGRATING ULTRASONIC WAVES WITH OPTICAL FIBER SENSORS}

\section{A. Interaction of Sensors With Acoustic Waves}

An acoustic wave can be defined in terms of a traveling pressure or particle velocity field where the pressure and velocity are related by the acoustic impedance as indicated in (1)

$$
\begin{aligned}
P(z, t) & =P_{o} \cos (w t-\beta z) \\
v(z, t) & =v_{o} \cos (w t-\beta z) \\
\frac{P_{o}}{v_{o}} & =Z_{A C} .
\end{aligned}
$$

The mechanical responses of an optical fiber are essentially to pressure fields (predominantly radial) or to strain fields (predominantly longitudinal). The strain field can be very easily derived from the velocity field, giving (2)

$$
\mathrm{E}(z, t)=\frac{2 \Pi v_{o}}{c_{p}} \cos (w t-\beta z)
$$


where $c_{p}$ is the phase velocity of the propagating ultrasound. Note that for a given power density the strain field is independent of frequency, while the displacement is inversely proportional to the acoustic frequency. Similarly the pressure field is also independent of frequency for a given acoustic power density. These relationships give valuable insight into the frequency response of optical fiber sensors.

In this paper, the main focus will be on the interaction between propagating ultrasound and either interferometric/polarimetric or Bragg grating sensors. The responses of interferometers/polarimeters are to integrate the strain or pressure field component which is responsible for changing the optical delay/birefringence along the fiber. The Bragg grating in contrast responds directly to strain provided that the strain is approximately uniform along the grating length. When the strain can no longer be considered to be uniform the response is a combination of spectral shift and spectral broadening which is in fact quite difficult to interpret unambiguously. We shall therefore concentrate on examining the overall integration of acoustic fields in interferometers and polarimeters and restrict our attention to the case of quasi homogenous strain for Bragg grating sensors.

It is important to note that the relationships in (1) and (2) apply strictly only to compression waves. The situation with shear waves is slightly different in replacing the pressure field with a shear stress. The relationships for hybrid waves such as Lamb and Rayleigh waves again shows the same trends but due deference must be made for the effects of mode shapes and mode fields on displacement and pressure perturbations. In these last more complex cases the mode field behavior can be utilized to optimize the performance of the fiber sensor though this will be beyond the scope of the current paper.

The ultrasonic responses of these sensors can now be predicted in broad terms. First of all, for integrating formats such as interferometers and polarimeters, the impact of the strain or pressure field to give integrated displacement or phase delay can be qualitatively assessed from straightforward spatial arguments. First take the extreme case of the sensor integration length being much longer than the acoustic/ultrasonic wavelength. This is fact the situation which applies for most practically based integrating sensors. If the integration along the length is analyzed, it is straightforward to demonstrate that, to a first approximation, this is a sinc function and so for the situation where the total length is more than a few wavelengths the net response is effectively zero. The net response is also by definition zero when the integration length is an integral number of wavelengths, provided that no significant attenuation occurs. This applies to the case of any parallel wave front with a propagation direction along the axis of the fiber. The situation for curved wave fronts is, as we shall see later, more complex since the amplitude of such wave fronts falls as the curvature deceases and the propagating energy diverges. Consequently, it is a safe starting assumption that when the integration length is much longer than the propagating ultrasonic wavelength the sensitivity of the sensor of ultrasonic waves propagating along its axis is very small.

Conversely, for waves propagating perpendicular to the axis of the fiber (that is wave fronts parallel to the optical fiber length) the sensor will integrate the interacting field along the fiber

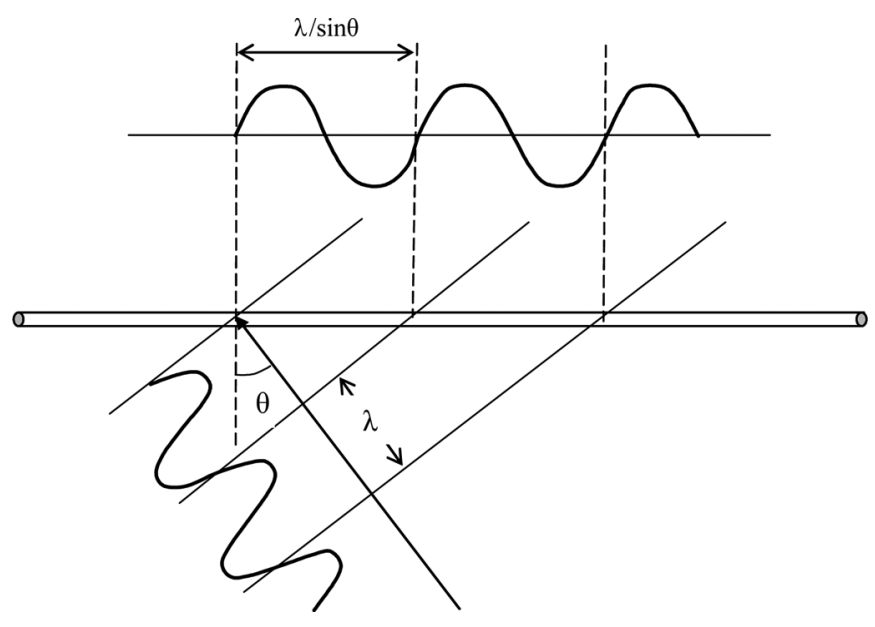

Fig. 1. Integration of a plane wave along a fiber sensor.

length. If the fiber is placed in a homogeneous compressional field then the pressure will be the parameter which influences total fiber delay. If the optical fiber is placed in an inhomogeneous pressure field (Lamb waves produce complex inhomogeneous pressure fields), this will impact upon the birefringence of the fiber and, as we shall see later can be utilized very effectively in a wave front integrating sensing system.

The contrasting case is that of the fiber Bragg grating sensor operating in a regime when the grating length (typically of the order of $1 \mathrm{~mm}$ ) is much less than the propagating acoustic wave length. Here it is the local strain field which modulates the grating reflection wave length. Interestingly then for a given acoustic propagation mode shape the frequency response of the fiber Bragg grating is independent of acoustic frequency over a very wide range. In order to scale these frequency responses, propagation velocity of a typical wave in solids is in the region of $5 \mathrm{~mm} / \mu$ s so that a $1 \mathrm{~mm}$ grating is significantly less than a wave length for frequencies up to around $500 \mathrm{kHz}$. This encompasses much of the frequency range which is of interest to the structural NDT community.

There are also many situations for which ultrasound can be detected using surface displacements especially in the out-ofplane direction. Variations on the theme of Michelson interferometers perform this function extremely well, though these differ from the other methods in that they do not use a sensing element bonded to the sample, but instead depends on analyzing the reflections of an optical beam from its surface. Indeed there are several commercial vibrometers which are available operating in this mode. This particular option will not be explored further in this paper, but it should be noted that these are very useful and very versatile instruments.

\section{B. Wave Front Integration}

The simplest case to analyze to show the effects of wavefront integration is that of a plane wave incident on a fiber sensor at an angle of $\Theta$ to the normal (Fig. 1). If an ultrasonic wavefront interacts with a straight optical fiber sensor, the phase of the wave, and hence the pressure acting on the sensor, will vary along its length. Assuming that the sensor response (typically changing effective refractive index or birefringence), is proportional to the 


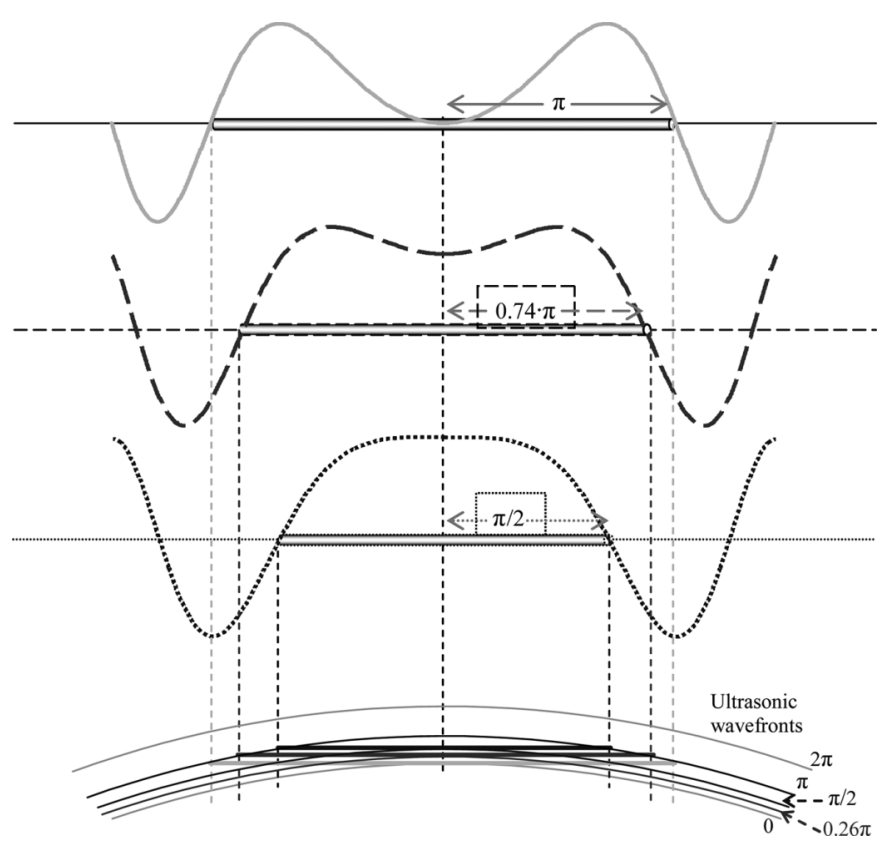

Fig. 2. Graphic demonstration of the optimal length for a fiber sensor to detect a cylindrical wavefront.

incident pressure, that response will be determined by the integrated value of the pressure along its length. Since the pressure changes produced by the wave as it traverses the fiber can be either positive or negative, there is clearly an optimum sensor length to source wavelength that can be employed if maximum sensitivity is to be obtained. In the case of a plane wave, if we neglect attenuation, then the optimum sensor length is clearly either that of half the acoustic wavelength resolved onto the fiber axis (i.e., $\lambda / 2 \sin \theta$ ), or this value multiplied by an odd-numbered integer.

In the majority of practical a plane wave front would be rarely encountered and it is necessary to analyze what happens when a curved wavefront interacts with a straight sensing fiber. To start with, consider the relatively simple case of an arc shaped wavefront with its propagation direction normal to the axis of the fiber (Fig. 2). Intuitively, it might be expected that the optimum sensor length would again be the longest over which the pressure could remain entirely positive (or negative). This would be the case if the wavefront phase difference between the center of the sensor and its end was $\pi$ radians as shown in the solid curve. The fact that this does not give the greatest integrated value is due to two factors.

i) The pressure magnitude is inversely proportional to the square root of the distance to the ultrasonic source (for a cylindrical wave). Therefore the pressure will be larger when it meets the center of the sensor than when it reaches a point further along for a similar wavefront phase value.

ii) The geometry of the relation between the arc of the wavefront and the straight fiber means that, for example, the length of the fiber sensor it interacts with, between the phases of 0 and $\pi / 2$ is longer than between $\pi / 2$ and $\pi$ (as shown in the wavefront arcs in the lower part of the figure).
It can be shown [7] that the optimum sensor length is, in fact that, for which a maximum phase difference of $0.74 \pi$ can occur between the center and the end of the sensor (dashed curve).

In Fig. 2, we have plotted three different situations in which the length of the sensor proportional to the distance form it to the ultrasound source gives.

1) Top (solid curve): a wavefront phase difference of $\pi$ between center and end of sensor.

2) Middle (dashed curve): a wavefront phase difference of $0.74 \pi$ between center and end of sensor.

3) Bottom (dotted curve): a wavefront phase difference of $\pi / 2$ between center and end of sensor.

Each case is based on the same normalization of the pressure wave, which is treated as a sine wave of unity amplitude and is inversely proportional to the square root square of the distance to the source. For the case plotted, the distance from the source to the center of sensor is taken as unity and 20 times greater than the ultrasonic wavelength.

The curves represent the pressure distribution along the different length sensors (in arbitrary units). It is obvious from this pictures the explanations above, how the case of $\pi$ radians phase difference (solid curve) integrates in most of the sensor length an almost zero value of the pressure field, therefore this explains how the integrated pressure of the dashed curve case $(0.74 \pi$ phase difference) integrates a much bigger pressure along its length.

The case of an acoustic wave whose propagation direction is at some arbitrary angle to the fiber is more complex and a detailed description is out of the scope of this paper. Analysis is carried out using the same basic principles; determining the amplitude and phase of the wave at each point along the fiber length and integrating over the appropriate part of its length. The center of this length will be the point at which the wave propagation vector intersects the axis of the fiber and integration will be over $\pm \mathrm{L} / 2$ about this point. The general trend will be the same as that predicted for a plane wave but with a ripple superimposed upon it, whose size and shape will be determined by source distance relative to the acoustic wavelength. Under certain circumstances it is possible that, for a given sensor length, the maximum sensitivity will not occur when the wave propagation direction is normal to the fiber (Fig. 3) [1].

\section{Lamb Waves}

Lamb waves are guided acoustic waves that propagate in sheets of material with their propagation vectors parallel to the surface. This characteristic, coupled with the fact that their energy is distributed throughout the sheet thickness, makes them an ideal candidate for use in structural health monitoring applications. One acoustic actuator can be used to generate a wave that can travel over, and thereby interrogate, a large area. It is the detection of these waves that will be the subject of the remainder of this paper. There are two types of wave, symmetric and antisymmetric, where the symmetry referred to is of the particle motion in the sheet relative to the neutral axis. This is shown in Fig. 4(a) and (b), [8] taken from an animation, from which it is clear that the modes contain both longitudinal and shear strains. 


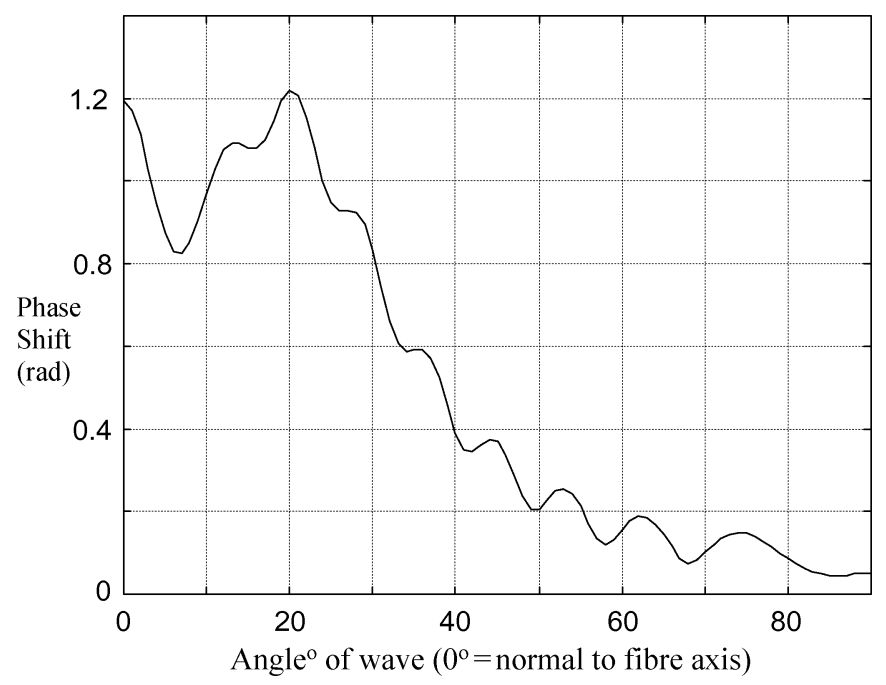

Fig. 3. Simulation of $\Delta \bar{\phi}$ peak-to-peak against the angle $\theta$ for aluminium at $270 \mathrm{kHz}$, (length of the sensor $=19.5 \mathrm{~cm}$; distance from the source to the center of the sensor $=16 \mathrm{~cm}$ ).
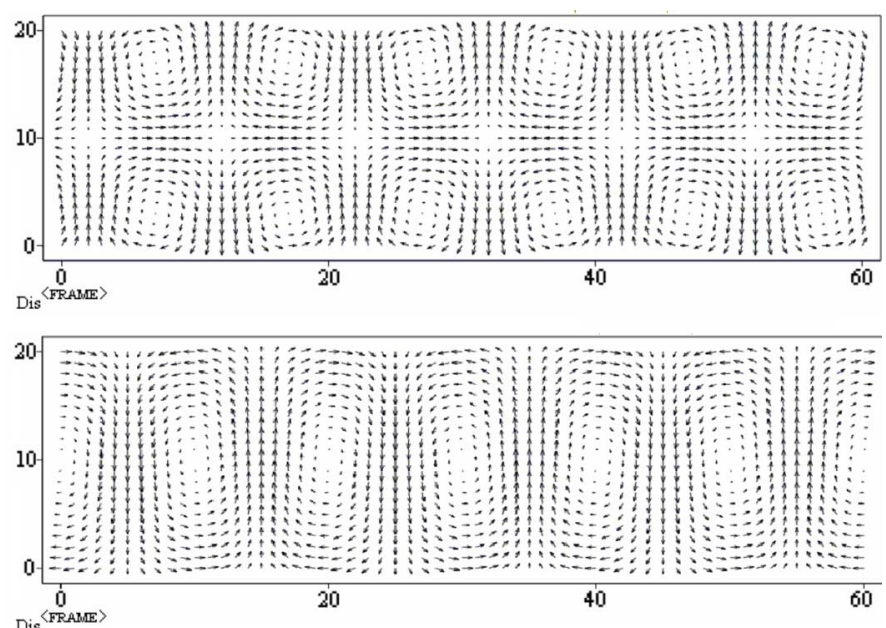

Fig. 4. Particle motion in (top) symmetric mode (bottom) antisymetric modes.

\section{Polarimetric Detection of LAMB Waves}

The first technique to be investigated for the detection of Lamb waves using a bonded fiber sensor was to use an interferometer to measure the changes in optical path length through the fiber produced by pressure induced changes in the fiber effective index [9]. Detailed examination of the results obtained using this method showed some unexpected polarization dependent effects that suggested the use of polarimetry as an alternative technique. Polarimetry is simple to implement and uses inexpensive components, therefore the experiment shown in Fig. 5 was set up. A linearly polarized laser source was connected via a polarization controller to the sensor fiber, which can be either attached to, or embedded in, the sample plate. It is simple to embed the fiber in a carbon fiber composite plate, but this is an anisotropic material and it was thought simplest to start with an isotropic material. Since bonding the fiber straight onto the surface of a sheet proved to give unreliable results, the fiber was cast into a small mould which was then bonded to the surface of

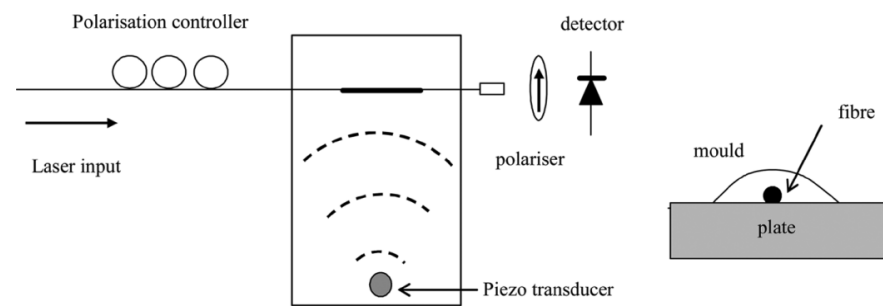

Fig. 5. Polarimetric detection of Lamb waves and cross section of fiber sensor moulding.

a Perspex sheet. The output of the sensor fiber was terminated with a GRIN lens, so that the light could be directed through a rotatable polarizer and on to a photodiode. Care was taken to ensure the output fiber was kept as short and as straight as possible so that the polarization state changed as little as possible between the end of the bonded length and the polarizer. The length of the bonded section of the fiber $(100 \mathrm{~mm})$ was chosen such that the acoustic wave was never more than $\lambda / 4$ out of phase along it. Lamb waves were generated by a piezoelectric transducer glued to the $3 \mathrm{~mm}$ thick plate and excited by a 5-cycle tone burst at the resonant frequency of the transducer/plate combination $(150 \mathrm{kHz})$. It was found that signals were produced by this set-up whose amplitudes were dependent both on the input state of polarization and the orientation of the polarizer. Accordingly, the following experiments were carried out.

i) A combination of the input polarization state and the polarizer orientation that gave the maximum signal amplitude was established. The polarizer was then rotated, while maintaining the same input polarization and the resulting changes in signal amplitude were measured, together with the dc power of the detected light. Measuring the minimum and maximum values of the de light level allowed the ellipticity and of the orientation of the major axis of the polarization state that gave the maximum signal amplitudes to be determined.

ii) Linear output states of polarization were set at various orientations by using the polarization controller to establish a null dc level in the orientation orthogonal to that to be studied. Signals were obtained by rotating the polarizer by $45^{\circ}$ and their amplitudes measured.

It must be emphasized at this point that, even in the absence of pressure from an acoustic wave, the output state of polarization would generally have been different to that at the input due to birefringence being produced in the fiber during the embedding or bonding processes.

The results of these experiments showed that the optimum output polarization state for maximum signal amplitude was an elliptical polarization state with a transmitted power aspect ratio of $2.64\left(\Rightarrow\right.$ E-field 1.62) at an orientation of $58^{\circ}$ with respect to the plane of the plate. The amplitude of the signal was 5\% of dc under optimum conditions, suggesting that the effects observed are sufficiently large to allow the technique to be used as a detection mechanism. Experiments conducted with linear output polarization showed little variation in signal amplitude with changing orientation of the polarization. The maximum signal obtained by using linear birefringence was less than $10 \%$ 

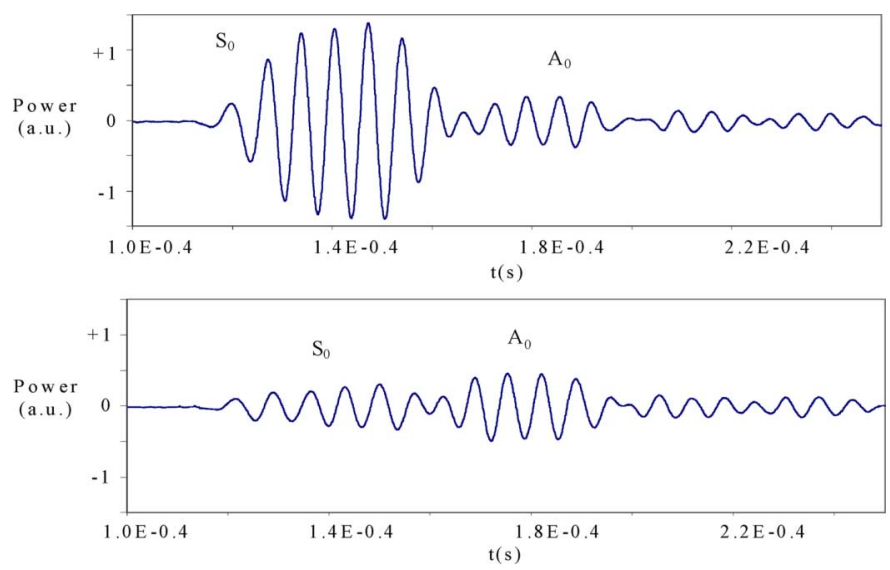

Fig. 6. Lamb wave signals obtained by the polarimetric method for different polarizer orientations: top $-10^{\circ}$ to the plane of the plate, bottom- $60^{\circ}$ orientation.

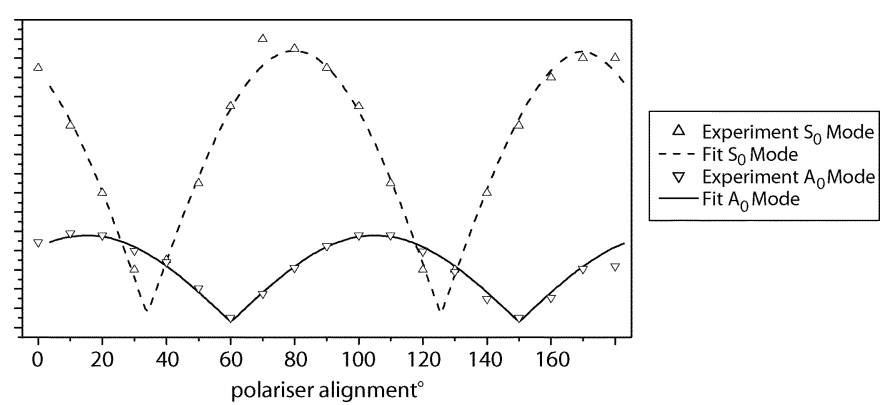

Fig. 7. Variation of $A_{o}$ and $S_{o}$ signal amplitudes with polarizer orientation (angles referenced to vertical).

of that obtained using the optimum polarization state. In the signals shown, the first pulse is the Lamb wave $S_{0}$, while the second is the slower asymmetric $A_{0}$.

It would be expected that, if the birefringence induced in the sensor fiber by the acoustic field had been purely linear, the output polarization state of the light required to give maximum signal amplitude would be circular. It would also be predicted that there would be an orientation of the linear light for which there would be zero signal amplitude, with a maximum signal amplitude being observed in the orthogonal direction. The combination of the above observations suggests that the birefringence induced by the acoustic wave is mainly linear, but with a smaller circular component. Although difficult to quantify, some idea of the possible origins of the circular birefringence may be gained by studying the particle motion of the Lamb waves as shown in Fig. 4(a) and (b). It is apparent that not only the velocity, but also the direction, of particle motion (and therefore the pressure field) varies with phase and, as stated earlier, the phase of the wave will vary slightly along the length of the fiber. The result of this will be that, at any given instant, the direction of the pressure gradient and hence the birefringence axes, will change along the length of the sensor. This rotation of the birefingent axis would impart a slight circular polarization to the fiber. It was also observed that the relative sensitivities of the $\mathrm{S}_{0}$ and $\mathrm{A}_{0}$ were to a considerable extent dependent on the orientation of the polarizer for a given output SOP (Figs. 6 and 7) and that other polarization states could be found for which the $\mathrm{A}_{0}$

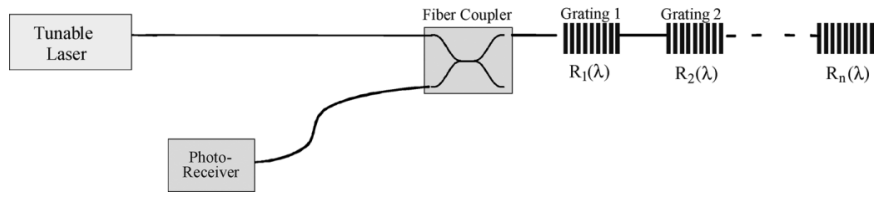

Fig. 8. Interrogation system for Bragg grating sensors.

signal was much larger than that for the $\mathrm{S}_{0}$. Presumably this is also due to the differences in pressure gradient modulation for the two modes. This effect has yet to be quantified, but suggests that more information could be obtained concerning the properties of the modes, such as the longitudinal and shear strain components, from polarimetric studies than from interferometry or a piezoelectric transducer alone. An obvious area of application would be to determine whether damage to a plate would cause detectable changes in the polarimetric properties of the signals. Experiments have also been carried out that demonstrate how the directional properties of the sensor can make acoustic reflections from a hole easier to detect by careful selection of the location of the source PZT relative to the fiber sensor [10].

\section{Fiber Bragg Grating Detection of LAMB WaVES}

The sensor reflection wave length from a Bragg grating is a direct measure of the optical path length corresponding to the grating periods. This in turn can be modulated by strain or pressure fields. The shift in wavelength is directly proportional to the strain (when it is assumed to be uniform) over the grating. If the strain is nonuniform the result is both a change in the average wavelength reflected and in the overall line width of the reflected spectrum. The nonuniform strain case while in principle tractable is in practice difficult to interpret. Although the strain along a grating length can never be perfectly uniform for the case of sinusoidally modulated strain, we can assume it to be effectively so, for the condition where the acoustic wavelength is far greater than the grating length. Consequently we shall concentrate on the uniform strain case. The Bragg grating, of course, also responds to pressure fields and this factor becomes increasingly relatively more important as the acoustic wave propagation direction becomes closer to normal to the fiber axis. Interestingly, for a given acoustic propagation mode shape the frequency response of the fiber Bragg grating is independent of acoustic frequency over a very wide range. In order to scale these frequency responses, the propagation velocity of a typical wave in a solid is in the region of $5 \mathrm{~mm} / \mu$ s so that a $1 \mathrm{~mm}$ grating is significantly less than a wave length for frequencies up to around $500 \mathrm{kHz}$. This encompasses much of the frequency range which is of interest to the structural NDT community.

The strain fields in typical ultrasonic waves are very smallat most microstrains and more typically nano strains and so the wavelength shifts are so small that the normal sensor detection schemes are ineffective. Consequently, we need to interrogate the FBG using a stabilized laser source (Fig. 8) which is capable of tracking changes in temperature or static strain, this being done by tuning the laser to the mid point of the linear portion of the FBG response curve. When the strain modulates the position of the curve on the wavelength axis, the reflected power will 


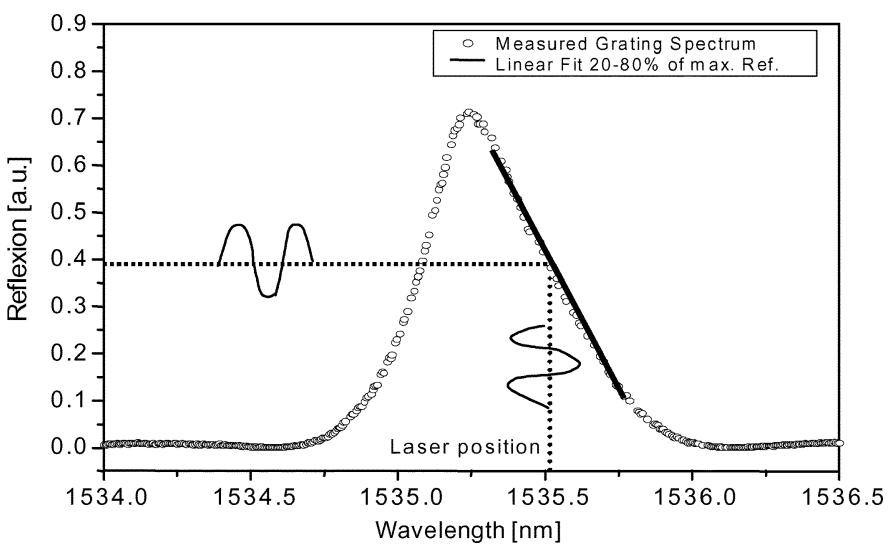

Fig. 9. Principle of FBG interrogation for ultrasound detection.

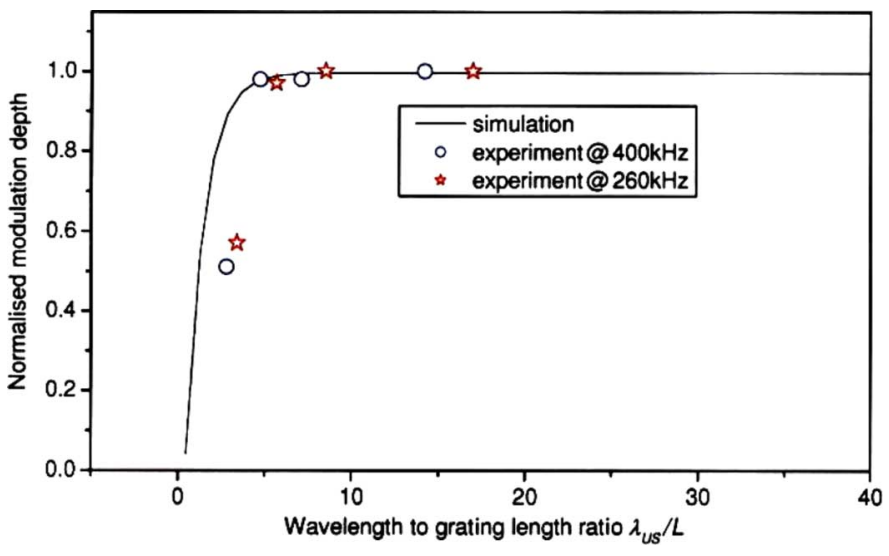

Fig. 10. Theoretical and experimental investigation of the effect of relative acoustic wavelength to sensitivity.

be similarly modulated (Fig. 9). The frequency response of this system (in addition to the acoustic wavelength/grating length ratio) is limited by the strain coupling between the sample and the fiber, which requires optimization of the recoating material and the bonding medium. The FBGs used were recoated with a tough Ormocer layer and bonded with a cyano-acrylate designed for strain gauge applications. The frequency response of the photodiode/amplifier system and the level of noise from the tunable laser are also crucial factors. With this system we can resolve dynamic strain fields with noise limited levels of the order of 100 picostrain [11].

Experiments to determine the practical effect of varying the grating length to acoustic wavelength ratio were carried out and show that above a ratio of about $1: 6$ the response is effectively independent of the acoustic signal wavelength (Fig. 10). The directional response of FBGs to ultrasound propagation direction (Fig. 11) was established by setting up an isotropic aluminium plate on which PZT source transducers were bonded in a semicircular array with a grating at the center. A reference PZT used as a detector was located close to the grating to allow normalization to be applied to the signals obtained from the individual source PZTs, which could vary due to differences in coupling. Each PZT was excited in turn and the amplitude of the signal obtained from the grating measured. As anticipated, the directional response characteristic was cosinusoidal

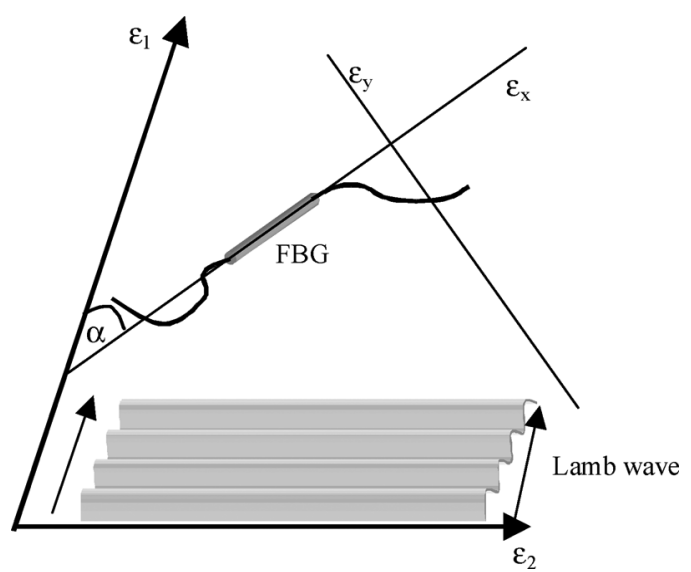

Fig. 11. Geometry for the description of FBG directional sensitivity.

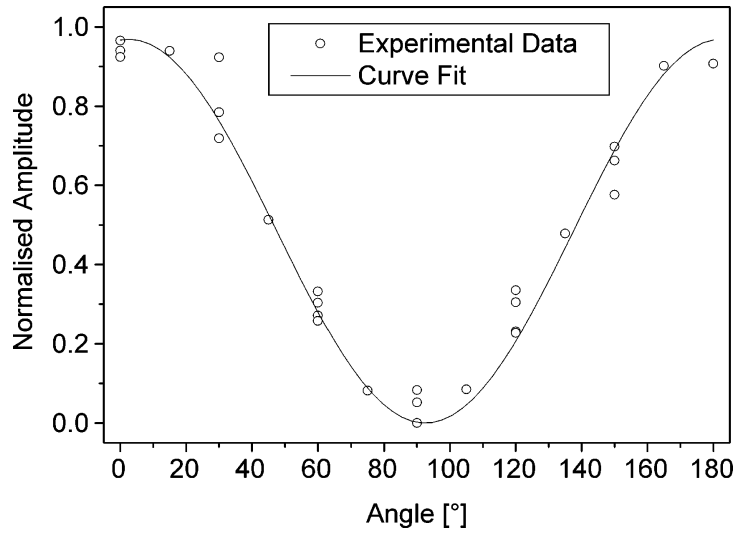

Fig. 12. Experimental and theoretical angular response of an FBG to an ultrasound wave.

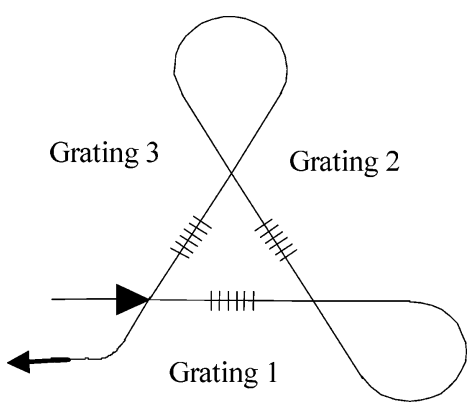

Fig. 13. Rosette configured from three FBGs.

(Fig. 12). Because the FBG has a length which is much shorter than the acoustic wavelength, it is not subject to the same wavelength integration issues that produce a complex angular response shape as the polarimeter, which requires a much longer length to achieve useful sensitivity. Note that the FBG, being primarily sensitive to strain, has its maximum sensitivity to an acoustic wave propagation direction along its axis, in contrast to the polarimeter, which is essentially sensitive to pressure and is therefore most sensitive to a wave direction normal to its axis.

The simple angular response of the FBG makes it suitable for detecting not only the amplitude, but also the direction, of an acoustic wave if three gratings of differing Bragg wavelength are configured into a rosette (Fig. 13). An additional grating can 


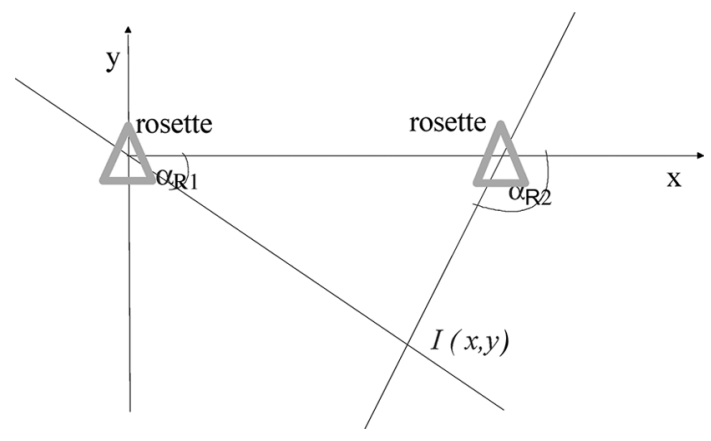

Fig. 14. Ultrasound source location using the intersection method.

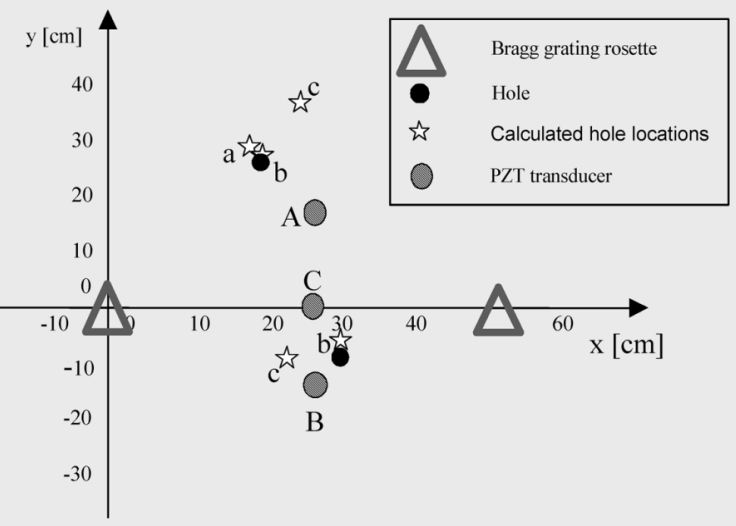

Fig. 15 Experimental determination of the locations of holes in an aluminium plate.

be included to measure or compensate for temperature changes. The basic method is similar to that in which electrical strain gauges are used to determine principle strains. The signal from the grating most nearly parallel to the acoustic wave direction will give the largest amplitude and the one closest to normal the smallest. The relative amplitudes of the signals are put into a suitable algorithm to determine both acoustic wave amplitude and direction. By using two rosettes the location of an acoustic wave can be determined by calculating the point of intersection of the directional vectors obtained from each (Fig. 14). Taking these ideas a stage further, if we consider a hole to be a passive source that can be excited by an active transducer such as a PZT, the method can be adapted to locate the position of a hole in a plate (Fig. 15). In order to do this we need to extract that part of the signal that is due to the presence of the hole. Measurements were taken from every grating in both rosettes, while being excited by each of the PZT transducers in turn. Excitation was carried out using a 5 cycle toneburst at $290 \mathrm{kHz}$ on a $1 \mathrm{~mm}$ thick aluminium plate. These parameters were chosen in order to ensure that only the $\mathrm{S}_{0}$ and $\mathrm{A}_{0}$ modes were excited and that these two modes were temporally separated. This was done both before and after the hole was produced in the plate and the signal due to the introduction of the hole was then obtained by subtracting the first set of signals from the second. This has been described in more detail in [12] and [13]. Fig. 15 shows the results of the comparison between the actual hole locations and those calculated from the signals obtained using each of three source PZTs in turn.
Another application of FBGs to detect Lamb waves has been the demonstration of their use in monitoring the growth of a crack during fatigue testing [14].

\section{CONCLUSION}

This paper has presented a brief overview of the principles of wave front integration and detection of ultrasonic waves using intrinsic optical fiber sensors. We show that relatively straightforward wavefront integration models can give useful predictions of both polar and frequency response functions for both long (compared to a wave length) and short interaction lengths. Sensors based on two different measurands, strain (FBGs) and pressure (fiber polarimeters) have been described and their responses compared. The principle of damage location using fiber Bragg grating rosettes to determine the direction of propagation of an incident ultrasound wave is also demonstrated. These concepts could readily be extended into more complex sensor networks. Fiber sensors offer advantages over conventional PZT sensors include their directional sensitivity, more broadband response, the ability to be multiplexed and the lack of a need for an electromagnetically sensitive, and frequently bulky wiring scheme.

\section{REFERENCES}

[1] N. E. Fisher, D. J. Webb, C. N. Pannell, D. A. Jackson, L. R. Gavrilov, J. W. Hand, L. Zhang, and I. Bennion, "Ultrasonic field and temperature sensor based on short in-fibre Bragg gratings," Electron. Lett., vol. 34, pp. 1139-1140, 1998.

[2] P. A. Fornitchov and S. Krishnaswamy, "Fibre Bragg grating ultrasound sensor for process monitoring and NDE applications," Rev. Progr. Quant. Nondestruct. Eval., vol. 21, pp. 937-944, 2002.

[3] N. Takahashi, S. Takahashiand, and K. Tetsumura, "Fibre Bragg grating underwater acoustic sensor," in Proc. 13th Int. Conf. Optical Fibre Sensors, 1999 , vol. 3746, pp. 565-568

[4] G. Coppola, A. Minardo, A. Cusano, G. Breglio, G. Zeni, A. Cutolo, A. Calabro, M. Guirdano, and L. Nicolais, "Analysis on the feasibility of the use of fiber Bragg grating sensors as ultrasound detectors," in Proc. Sensory Phenomena and Measurement Instrumentation for Smart Structures and Materials, 2001, vol. 4328, pp. 224-232.

[5] H. Tsuda, N. Toyama, K. Urabe, and J. Takatsubo, "Impact damage detection in CFRP using fiber Bragg gratings," Smart Mater. Struct., vol. 13, pp. 719-724, 2004.

[6] N. Takeda, Y. Okabe, J. Kuwahara, S. Kojima, and T. Ogisu, "Development of smart composite structures with small-diameter fiber Bragg grating sensors for damage detection: Quantitative evaluation of delamination length in CFRP laminates using Lamb wave sensing," Comp. Sci. Tech., vol. 65, pp. 2575-2587, 2005, 2005.

[7] B. Sorazu, G. Thursby, B. Culshaw, F. Dong, Y. Yong, and J. Yao, "Ultrasound wavefront integration using optical fibre sensors," in Proc SPIE Smart Structures and Materials, San Diego, CA, 2003, vol. 5050, pp. 23-33.

[8] V. Giurgiutiu, J. Bao, and W. Zhao, "Active sensor wave propagation health monitoring of beam and plate structures," in Proc. 8th Int. Symp. Smart Structures and Materials, Newport Beach, CA, 2001.

[9] S. G. Pierce, W. R. Philp, B. Culshaw, A. Gahagan, A. McNab, G. Hayward, and F. Lecuyer, "Surface bonded optical fibre sensors for the inspection of CFRP plates using ultrasonic Lamb waves," Smart Mater. Struct., vol. 5, pp. 776-787, 1996.

[10] G. Thursby, B. Sorazu, F. Dong, D. Betz, and B. Culshaw, "Damage detection in structural materials using a polarimetric fiber optic sensor," in Proc. SPIE Smart Structures and Materials, San Diego, CA, 2003, vol. 5050, pp. 61-70.

[11] D. C. Betz, G. Thursby, B. Culshaw, and W. J. Staszewski, "Acoustoultrasonic sensing using fiber Bragg gratings," Smart Mater. Struct, vol. 12, pp. 122-128, Feb. 2003.

[12] D. C. Betz, G. J. Thursby, B. Culshaw, and W. J. Staszewski, "Structural damage identification using multifunctional Bragg grating sensors-Part I: Theory and implementation," J. Smart Mater. Struct., vol. 15, no. 5, pp. 1305-1312, Oct. 2006. 
[13] D. C. Betz, W. J. Staszewski, G. J. Thursby, and B. Culshaw, "Structural damage identification using multifunctional Bragg grating sensors-Part II: Damage detection results and analysis," J. Smart Mater. Struct., vol. 15, no. 5, pp. 1313-1322, Oct. 2006.

[14] D.C. Betz, G. J. Thursby, B. Culshaw, and W. J. Staszewski, "Multifunctional fibre Bragg grating sensors for crack detection in metallic structures," J. Aerosp. Eng., vol. 220, no. 5, pp. 453-461, Jan. 2006.

Brian Culshaw was born in Lancashire, U.K., on September 24, 1945. He graduated in physics from University College London (UCL), London, U.K., in 1966 and received the Ph.D. degree in electronic and electrical engineering (specializing in microwave semiconductors) in 1969.

After a year at Cornell University, he joined Bell Northern Research (now Nortel), Ottawa, ON, Canada, and while continuing to work on microwave semiconductors, developed an interest in fiber optic technology. Late in 1973, he returned to UCL and, after two further years as a post-doc working on semiconductor device simulation, developed his interest in fiber optic sensor technologies, their principles, and applications. His research has encompassed fiber gyroscopes, hydrophones, spectroscopic analysis systems, and mechanical interferometric sensors. In 1983, he became Professor of optoelectronics at Strathclyde University, Glasgow, U.K. In the mid-1980s, he was the founding editor for the International Journal of Optoelectronics, and until mid 2004 was a topical editor for Applied Optics. He has edited for over a decade with Alan Rogers of Surrey University the Artech House series in optoelectronics (now over 50 titles). He has administered several major research initiatives, particularly multipartner EU programmes in sensing, measurement, fiber optics, and smart structures. He has reviewed research activities and proposals in the U.K. and elsewhere. He has also acted internationally in Ph.D. and Habilitation examinations.

Dr. Culshaw was de facto technical chair of the first (1983) International Conference on Optical Fibre Sensors (OFS), now a series regarded as the definitive meeting in the community. He chaired the tenth in Glasgow and was technical co-chair of the 17th in Bruges in 2005. He orchestrated with SPIE the CD-ROM of the series proceedings which has recently been reissued. He also initiated European meetings in smart structures and the EWOFS workshop series in optical fiber sensor technology. Predominantly with SPIE, he has organized numerous other conferences and workshops in Europe the U.S. and Asia. He was the 2007 President of SPIE.

Graham Thursby, photograph and biography not available at the time of publication.

Daniel Betz, photograph and biography not available at the time of publication.

Borja Sorazu, photograph and biography not available at the time of publication. 